\title{
UNIQUE ISOMETRIC IMMERSION INTO HYPERBOLIC SPACE
}

\section{BELTAGY}

\author{
Mathematics Department \\ Faculty of Science \\ Tanta University \\ Egypt
}

(Received February 6, 1992 and in revised form August 24, 1992)

\begin{abstract}
A result concerning equivalence of isometric immersions with related Gauss maps into hyperbolic space has been established.
\end{abstract}

KEY WORDS AND PHRASES. Isometric immersion, rigidity, second fundamental tensor, Lorentz manifold, Minkowski space.

1991 AMS SUBJECT CLASSIFICATION CODES. 53C40, 53C42.

\section{INTRODUCTION.}

Equivalence between isometric immersions of a Riemannian manifold into another one has been an interesting fruitful area of research for a long time ([1], [4], [6], [7], [12]). The concept of equivalence between two isometric immersions may be given as follows.

Let $f_{1}: M \rightarrow N$ and $f_{2}: M \rightarrow N$ be two different isometric immersions of a Riemannian manifold $M$ into another Riemannian manifold $N$. The immersion $f_{1}$ will be equivalent to $f_{2}$ (up to an isometry $\tau$ and $N$ ) if there exists an isometry $\tau: N \rightarrow N$ such that $\tau \circ f_{1}=f_{2}$. We may write $f_{1}=f_{2}$ (mod. $r)$.

If the above mentioned concept is satisfied for each pair of isometric immersions of $M$ into $N$, we say that $M$ is uniquely isometrically immersed in $N$. In classical differential geometry $M$ is said to be rigid.

If for each pair of isometric immersions $f_{1}: M \rightarrow N$ and $f_{2}: M \rightarrow N^{\prime}$ there exists a continuous curve $\tau_{s}, s \in[0, a]$ in the group $G$ of isometries of $N$ such that $\tau_{o}=i d$ and $\tau_{a} \circ f_{1}=f_{2}$, we say that $M$ is uniquely continuously isometrically immersed into $N[8]$.

In [1] the following remarkable result concerning isometric immersions into $(n+1)$-Euclidean space $E^{n+1}$ has been proved.

THEOREM 1.1. Let $M$ be a connected, orientable $C^{\infty}$-Riemannian $n$-manifold. Suppose that the Gauss maps of each pair $f$ and $\bar{f}$ of isometric immersions of $M$ into $E^{n+1}$ differ by an isometry of $E^{n+1}$. Then $M$ is uniquely isometrically immersed in $E^{n+1}$ if any of the following conditions is satisfied.

(a) $M$ is compact,

(b) There exists at least one point $m \in M$ where all sectional curvatures are positive,

(c) There exists at least one point $m \in M$ such that $f$ (or $\bar{f}$ ) is not minimal at $m$, and $M$ has no flat points with respect to $f$ (or $\bar{f}$ ). 
As far as we are concerned, the corresponding study in hyperbolic space $H^{n+1}$ has not yet been considered. Accordingly, we devote the present work to deal with the rigidity problem of hypersurfaces in hyperbolic space with constant sectional curvature -1 as a receiving space. The main result will be stated in the next section. From now on all manifolds and maps are sufficiently smooth for computations to make sense.

2. PRELIMINARIES AND RESULTS.

We begin this section by giving a brief word about the model of hyperbolic space we shall use throughout the paper.

In $\mathbf{R}^{n+2}$ with the natural basis $e_{0}, e_{1}, e_{2}, \cdots, e_{n+1}, n \geq 1$, we consider a nondegenerate symmetric bilinear form

$$
b(x, y)=-x_{o} y_{o}+\sum_{k=1}^{n+1} x_{k} y_{k} \quad x, y \in \mathbf{R}^{n+2} .
$$

The pair $V^{n+2}=\left(\mathbf{R}^{n+2}, b\right)$ is called the Minkowski space.

Let $0(1, n+1)$ denote the orthogonal group of $b$, i.e.,

$$
\begin{aligned}
0(1, n+1) & =\{A \in G 1(n+2, R): b(A x, A y)=b(x, y)\} \\
& =\left\{A \in G 1(n+2, R): A^{\prime} S A=S\right\},
\end{aligned}
$$

where

$$
S=\left[\begin{array}{cc}
-1 & 0 \\
0 & I_{n+1}
\end{array}\right]
$$

The fact that $A^{\prime} S A=S$ implies that $\operatorname{det} A= \pm 1$. A matrix $A=\left(a_{i j}\right)$ in $0(1, n+1)$ belongs to the identity component if and only if $\operatorname{det} A=1$ and $a_{o o}>1$. Let $G$ denote the identity component of $0(1, n+1)$.

Now, the hypersurface $\widetilde{M}$ in $V^{n+2}$ defined by $b(x, y)=-1$ is the disjoint union of two connected components:

$$
H^{n+1}=\left\{x \in \widetilde{M}: x_{o} \geq 1\right\} \quad \text { and } \quad K^{n+1}=\left\{x \in \widetilde{M}: x_{o} \leq-1\right\} .
$$

$0(1, n+1)$ acts transitively on $\widetilde{M}$ while $G$ acts transitively on $H^{n+1}[9]$.

Let $x \in H^{n+1}$, i.e., $b(x, y)=-1$ and $x_{o} \geq 1$. The tangent space $T_{x} H^{n+1}$ is given, through the identification by parallel displacement in $V^{n+2}$, by the subspace of all vectors $a \in V^{n+2}$ such that $b(x, a)=0$. The restriction of $b$ to $T_{x} H^{n+1}$ is positive definite. Thus the form $b$ restricted to the tangent space at each point of $H^{n+1}$ gives rise to a complete Riemannian metric on $H^{n+1}$ which is obviously invariant under $G$. Moreover, $\left(H^{n+1}, b\right)$ is of constant sectional curvatures $K=-1$. Hence $H^{n+1}$ will be taken to be the model in which our immersions occur. For geodesics, horodiscs, horospheres, central projection and other geometric properties of $H^{n+1}$ we refer the reader to $([4],[14])$. 
The hypersurface $\widetilde{U}^{n+1}$ in $V^{n+2}$ defined by $b(x, y)=1$ is connected Lorentz. We call $\widetilde{H}^{n+1}$ the conjugate hypersurface of both $H^{n+1}$ and $k^{n+1}$. (See Fig. 1).

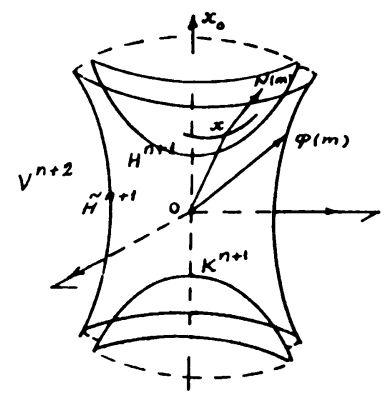

Figure 1

Let $f: M \rightarrow H^{n+1}$ be an immersion of the orientable manifold $M$. The Gauss map $\phi: M \rightarrow \widetilde{H}^{n+1}$ of this immersion is defined to be the parallel translation of the unit normal $N(m)$ of $M$, as a hypersurface of $H^{n+1}$, at $f(m)$ up to the origin 0 of $V^{n+2}$. Hence, the Gauss image of $M$ will lie on the conjugate hypersurface $\widetilde{H}^{n+1}$ of $H^{n+1}$.

Now, we state our result.

THEOREM 2.1. Let $f$ and $\bar{f}$ be two $C^{\infty}$ isometric immersions of a connected, orientable Riemannian $n$-manifold $M(n \geq 2)$ into the $(n+1)$-hyperbolic space $H^{n+1} \subset V^{n+2}$. Suppose that the Gauss maps $\phi$ and $\bar{\phi}$ differ by a matrix $\sigma \in G$. Then with any one of the conditions (i)-(iii) below, $f$ and $\bar{f}$ differ by an isometry $\tau$ of $H^{n+1}$, i.e., $\tau \circ \bar{f}=f$.

(i) $M$ has at least one point $m \in M$ where all sectional curvatures are greater than -1 .

(ii) $M$ is compact.

(iii) There exists at least one point $m \in M$ such that $f$ (or $\bar{f}$ ) is not minimal at $m$, and there is no point in $M$ with $A=0$ (or $\bar{A}=0$ ), where $A$ is the second fundamental tensor.

Assume that $\bar{\phi}=\sigma \circ \phi$ where $\sigma$ is an element of $G$, then we can show that the immersion $\overline{\bar{f}}=\sigma \circ f: M \rightarrow H^{n+1}$ has the Gauss map $\overline{\bar{\phi}}$ of the form $\overline{\bar{\phi}}= \pm \sigma \circ \phi$. The sign depends on whether $\sigma$ is orientation preserving or not. Hence $\bar{\phi}= \pm \phi$ which will be used, just for simplicity, instead of $\bar{\phi}=\sigma \circ \phi$.

In the light of the above discussions, let us take $\bar{\phi}=\phi$ and notice that if $\bar{\phi}=-\phi$ we may change the orientation to have again $\bar{\phi}=\phi$. In this way, $N(m)=\bar{N}(m)$ for every $m \in M$ up to a parallel translation in $V^{n+2}$. Let $D$ be the Levi-Civita connection of $V^{n+2}$ and $\tilde{\nabla}$ be the induced connection on $H^{n+1}$. Applying Weingarten equation to $f(M)$ and $\bar{f}(M)$ as immersed hypersurfaces of $H^{n+1}$, we have

$$
\begin{array}{rl}
\tilde{\nabla}_{f_{*} X} & N=f_{*}(-A X), \\
\tilde{\nabla}_{\bar{f}_{*} X} & \bar{N}=\bar{f}_{*}(-\bar{A} X),
\end{array}
$$

where $A$ and $\bar{A}$ are the second fundamental tensors of $f$ and $\bar{f}$, respectively, and $X$ is a tangent vector of $M$. If we write $D_{f_{*} X} N$ in terms of its tangent component to $H^{n+1}$ and the normal one, we have

$$
D_{f_{*} X} N=\widetilde{\nabla}_{f_{*} X} N+\mu f(x)
$$

Since $b\left(N, f_{*} X\right)=0$, then

$$
f_{*} X b(N, f(x))=b\left(D_{f_{*} X} N, f(x)\right)+b\left(N, f_{*} X\right)=0 \Rightarrow
$$




$$
\begin{aligned}
& b\left(D_{f_{*}} X\right.N, f(x))=0 \\
& \mu=0 .
\end{aligned}
$$

To be consistent with the references we depend on, we shall replace $b(X, Y)$ by $\langle X, Y\rangle$ in all the following computations.

From (2.2a) and (2.2b), we have

$$
\begin{aligned}
D_{f_{*} X} & =\tilde{\nabla}_{f_{*} X} N \\
D_{\bar{f}_{*} X} \bar{N} & =\widetilde{\nabla}_{\bar{f}_{*} X} \bar{N}
\end{aligned}
$$

From equations (2.1)-(2.3) we have

$$
\begin{array}{rl}
D_{f_{*} X} & N=f_{*}(-A X), \\
D_{\bar{f}_{*} X} & \bar{N}=\bar{f}_{*}(-\bar{A} X)
\end{array}
$$

As $N=\bar{N}$, then equations (2.4) give

$$
f_{*}(A X)=\bar{f}_{*}(\bar{A} X)
$$

up to a parallel translation in $V^{n+2}$.

LEMMA 2.1. $A$ and $\bar{A}$ have the same null space at each point of $M$.

PROOF. It is sufficient to show that $A X=0$ if and only if $\bar{A} X=0$. If $A X=0$, then $f_{*}(A X)=0$ and so $\bar{f}_{*}(\bar{A} X)=0$. Since $\bar{f}$ is an immersion, then $\bar{f}_{*}$ is injective and hence $\bar{A} X=0$. The converse is direct.

LEMMA 2.2. $A^{2}=\bar{A}^{2}$.

PROOF. For arbitrary vectors $X$ and $Y$ tangent to $M$, we have

$$
\begin{aligned}
\left\langle\bar{A}^{2} X, Y\right\rangle & =\langle\bar{A} X, \bar{A} Y\rangle=\langle\bar{f} *(\bar{A} Y)\rangle \\
& =\left\langle f_{*}(A X), f_{*}(A Y)\right\rangle=\langle A X, A Y\rangle=\left\langle A^{2} X, Y\right\rangle .
\end{aligned}
$$

Hence the result.

LEMMA 2.3. $(\operatorname{tr} A) A=(\operatorname{tr} \bar{A}) \bar{A}$

PROOF. The Ricci tensor $S$ of $M$, as being preserved by isometries, may be written as [9]

$$
S=\widetilde{S}+(\operatorname{tr} A) A-A^{2}=\widetilde{S}+(\operatorname{tr} \bar{A}) \bar{A}-\bar{A}^{2}
$$

where $\widetilde{S}$ is the Ricci tensor of $H^{n+1}$. Then

$$
(\operatorname{tr} A) A-A^{2}=(\operatorname{tr} \bar{A}) \bar{A}-\bar{A}^{2} .
$$

Using Lemma 2.2, we obtain the required result.

\section{LEMMA 2.4.}

(i) $\quad(\operatorname{tr} A)^{2}=(\operatorname{tr} \bar{A})^{2}$

(ii) If $\operatorname{tr} A \neq 0$ at $m$, then $\vec{A}= \pm A$ at $m$.

(iii) The choice of the sign in (ii) is constant in each component of the open set $V=\{m \mid(\operatorname{tr} A)(m) \neq 0\}$.

PROOF. Part (i). This becomes direct from Lemma 2.3 when taking the trace of both sides of equation (2.7).

Part (ii). If $(\operatorname{tr} A)(m) \neq 0$, then by part (i) we have that $(\operatorname{tr} \bar{A})(m) \neq 0$. Using equation (2.8), we have $[(\operatorname{tr} A) /(\operatorname{tr} \bar{A})](m)= \pm 1$. Applying equation (2.7), we obtain that $\bar{A}= \pm A$ at $m$.

Part (iii). If $(\operatorname{tr} A)(m) \neq 0$, then as $A$ is a continuous tensor field we have that $(\operatorname{tr} A) \neq 0$ near 
$m$. Hence the subset $V=\{m \mid(\operatorname{tr} A)(m) \neq 0\}$ is an open subset of $M$.

Now consider the following cases:

(a) Let $A=\bar{A}$ at $m$ and assume that in each neighborhood $U_{i}$ of $m$ there exists a point $m_{i}$ where $A\left(m_{i}\right)=-\bar{A}\left(m_{i}\right)$. In this way, we obtain a sequence of points $\left\{m_{i}\right\}$ for which $A\left(m_{i}\right)=-\bar{A}\left(m_{i}\right)$. Taking the limit of both sides as $m_{i} \rightarrow m$ we have

$$
\lim _{m_{i} \rightarrow m} A\left(m_{i}\right)=-\lim _{m_{i} \rightarrow m} \bar{A}\left(m_{i}\right)
$$

By the continuity of both $A$ and $\bar{A}$, we have $A(m)=-\bar{A}(m)$ which contradicts the assumption and so $A=\bar{A}$ near $m$.

(b) If $A=-\bar{A}$ at $m$, we follow a similar discussion.

\section{A RIGIDITY RESULT.}

In this section we give a general rigidity result for hypersurfaces in $H^{n+1}$ similar to Theorem 6.4 p. 45-46 [9]. For notations and details we refer to [9]. Although the following result has been already given in [13], we write its full proof as it is too important for the subsequent results.

THEOREM 3.1. Let $M$ be a connected $n$-dimensional Riemannian manifold and let $f$ and $\bar{f}$ be isometric immersions of $M$ into $H^{n+1} \subset V^{n+2}$ with fields of unit normals $\xi$ and $\bar{\xi}$, respectively. If the second fundamental forms $h$ and $\bar{h}$ of $f$ and $\bar{f}$ (with respect to $\xi$ and $\bar{\xi}$ ), respectively, coincide on $M$, then there is an isometry $r$ of $H^{n+1}$ such that $\tau \circ \bar{f}=f$.

PROOF. We follow the same procedure of [9] and start with the local version of the theorem.

Assume that $x_{o}$ is a point of $M$. We have two different frames $\left(e_{1}, e_{2}, \cdots, e_{n}, \xi, y\right)$ and $\left(\bar{e}_{1}, \bar{e}_{2}, \cdots, \bar{e}{ }_{n}, \bar{\xi}, \bar{y}\right)$ at $x_{o}$, where $y=f(x)$ and $\bar{y}=\bar{f}(x)$, respectively, such that

$$
\begin{gathered}
\left\langle e_{i}, e_{j}\right\rangle=\left\langle\bar{e}_{i}, \bar{e}_{j}\right\rangle \quad 1 \leq i, j \leq n \\
\left\langle\xi, e_{i}\right\rangle=\left\langle\bar{\xi}, \bar{e}_{i}\right\rangle=0, \quad\left\langle y, e_{i}\right\rangle=\left\langle\bar{y}, \bar{e}_{i}\right\rangle=0, \quad\langle y, \xi\rangle=\langle\bar{y}, \bar{\xi}\rangle=0 .
\end{gathered}
$$

As $G$ acts transitively on $H^{n+1}$, then there exists an orthogonal matrix $\tau \in G$ which is an isometry of $H^{n+1}$ such that $\tau$ maps the frame $\left(e_{1}, e_{2}, \cdots, \xi, y\right)$ at $x_{o}$ upon the frame $\left(\bar{e}_{1}, \bar{e}{ }_{2}, \cdots, \bar{\xi}, \bar{y}\right)$ at the same point $x_{o}$.

In terms of local coordinates in a coordinate neighborhood $U$ about $x_{o}$, simple calculations using Gauss and Weingarten equations give the following two systems of partial differential equations

$$
\left.\left.\begin{array}{l}
\frac{\partial e_{j}}{\partial x_{i}}=g_{i j} y+\sum_{k} \Gamma_{i j}^{k} e_{k}+h_{i j} \\
\frac{\partial \xi}{\partial x_{j}}=-\sum_{k} a_{j}^{k} e_{k}
\end{array}\right\}(I) \quad \begin{array}{l}
\frac{\partial \bar{e} j}{\partial x_{i}}=g_{i j} \bar{y}+\sum_{k} \Gamma_{i j}^{k} \bar{e}_{k}+h_{i j} \\
\frac{\partial \bar{\xi}}{\partial x_{j}}=-\sum_{k} a_{j}^{k} \bar{e}_{k}
\end{array}\right\}
$$

The main difference between these two systems and those of [9] is that the systems here are nonhomogeneous. In spite of this difference, the existence and uniqueness principle of solutions is still working.

Since the two systems $(I)$ and $(\bar{I})$ above have the same initial conditions at $x_{o}$, then by uniqueness we have

$$
e_{j}=\bar{e}, \quad \xi=\bar{\xi} \quad \text { on } U, \quad 1 \leq j \leq n
$$


As $e_{j}=\partial y / \partial x_{j}, \quad \bar{e}{ }_{j}=\partial \bar{y} / \partial x_{j}$, and since $y\left(x_{o}\right)=\bar{y}\left(x_{o}\right)$, we have that $y=\bar{y}$ on $U$ and the local version is now proved.

The global version of the above theorem can be proved exactly as that of immersions into Euclidean space $E^{n+1}[9]$.

Notice that for immersions into Euclidean space $E^{n+1}$, the required isometry is a resultant of translation in addition to an orthogonal matrix $r \in 0(n+1)$. From the above theorem, it becomes clear that in the case of immersions into hyperbolic space $H^{n+1}$ the isometry needed to establish the proof is obtained purely from an orthogonal matrix of $G$ acting on $H^{n+1}$ by restriction.

\section{PROOF OF THEOREM 2.1.}

In the light of Theorem 3.1, if we consider $V_{1}$ to be the component of $V$ on which $\bar{A}=A$, then there exists an isometry $\tau$ of $H^{n+1}$ such that $\tau \circ \bar{f}=f$ on $V_{1}$. In the following we given more significance of $\tau$.

Let us consider a point $m \in V_{1}$ and take $X \in T_{m} M$ to be an eigenvector of $A(=\bar{A})$ corresponding to a non-zero eigenvalue. Then equation (2.5) gives

$$
f_{*}(X)=\bar{f}_{*}(X)
$$

Consequently, if $(\operatorname{rank} A)(m)=n$, then all the eigenvalues of $A$ are non-zeros and so $f_{*}\left(T_{m} M\right)=\bar{f}_{*}\left(T_{m} M\right)$ up to a parallel translation in $V^{n+2}$. Now consider the following two different cases:

Case (i). $\quad \bar{f}(m)=f(m), \bar{f}_{*}\left(T_{m} M\right)=f_{*}\left(T_{m} M\right)$ and $\bar{N}(m)=N(m)$.

In this case, the needed isometry of $H^{n+1}$ turns out to be the identity map.

Case (ii). $\quad f(m) \neq \bar{f}(m), \bar{f}_{*}\left(T_{m} M\right) / / f_{*}\left(T_{m} M\right)$ and $\bar{N}(m) / / N(m)$.

Clearly, $\bar{f}(m)$ and $f(m)$ should be antipodal points of $\widetilde{M}$. This situation will not occur unless $f(M) \subset H^{n+1}$ and $\bar{f}(M) \subset K^{n+1}$ which contradicts the assumption that both of the immersions $f$ and $\bar{f}$ are in $\boldsymbol{H}^{n+1}$. Consequently, this case should be disregarded.

Using Theorem 3.1, we have the following.

LEMMA 4.1 If $V_{1}$ is a component of $V$ where $\bar{A}=A$ and $\operatorname{rank} A=n$ at one point $m \in V_{1}$ then $f(x)=\bar{f}(x)$ on $V_{1}$.

On the other hand if $A=-\bar{A}$ and $(\operatorname{rank} A)(m)=n$, then $f_{*}(X)=-\bar{f}_{*}(X) \forall X \in T_{m} M$.

In this case $f_{*}\left(T_{m} M\right)$ will also be parallel to $\bar{f}_{*}\left(T_{m} M\right)$ and as mentioned above $f(m)$ should coincide with $\bar{f}(m)$. The isometry of $H^{n+1}$ needed is different from the identity. In fact it is some sort of reflection.

LEMMA 4.2. If $V_{2}$ is a component of $V$ where $\bar{A}=-A$ and rank $A=n$ at one point $m \in V_{2}$ then the matrix $\tau \in G$ which satisfies $\tau(m)=m, \tau(\bar{N})=N$ and $\tau(X)=-X \forall X \in T_{m} M$ has the property that $\tau \circ \bar{f}(x)=f(x)$ on $V_{2}$.

In the following, we shall restrict ourselves to the case of Lemma 4.1 mentioned above and give a hint to show how to deal with the case of Lemma 4.2.

Let us define the real-valued function $F$ on $M$ as follows

$$
F(x)=\langle f(x), c\rangle,
$$

where $c$ is a constant vector. Following computations similar to that in $([9]$, p. 342$)$ we have that

or

$$
\Delta F=n F+n\langle\eta, c\rangle,
$$




$$
\widetilde{\Delta} \boldsymbol{F}=n\langle\eta, c\rangle,
$$

where $\widetilde{\Delta}=\Delta-n$ and $\eta$ is the mean curvature vector $\eta=\frac{1}{n}(\operatorname{tr} A) N$ of $M$ as a hypersurface of $H^{n+1}$. The new operator $\widetilde{\Delta}$ is elliptic and has $C^{\infty}$ coefficients.

We are now in a position to prove Theorem 2.1.

PROOF OF PART (i). Let $m$ be a point of $M$ where all sectional curvatures are greater than -1 . Then $\operatorname{rank} A=\operatorname{rank} \bar{A}=n$ and $A$ is definite at $m$. Let $m \in V_{1}$ where $V_{1}$ is the component of $V$ where $\bar{A}=A$. Hence $\bar{f}_{*}(X)=f_{*}(X)$ for each tangent vector $X \in T_{m} M$. From the above discussions, the identity map $I$ will be the required isometry which takes $\bar{f}\left(V_{1}\right)$ to $f\left(V_{1}\right)$.

Let $W$ be the largest open set of $M$ containing $V_{1}$ such that $\bar{x}=x$. Following the same procedures of [1] we conclude that for every boundary point $p \in \partial W$, there exists a neighborhood $Q$ of $p$ such that for each $q \in Q \cap V, \bar{A}=A$ at $q$ which means that $Q$ intersects the component $V_{1}$ only. Hence we may write $W \cup Q \subset V_{1} \cup \bar{V}_{2}$. For points in $V_{1}$, we have $\bar{\eta}=\eta$ as $\bar{A}=A$ on $V_{1}$, and for points in $Q-V_{1}$, we have $\bar{\eta}=\eta=0$. Consequently, $\bar{\eta}=\eta$ on the whole of $W \cup Q$.

Consider the real-valued function $g: W \cup Q \rightarrow R$ defined by

$$
g(x)=\langle f(x)-\bar{f}(x), c\rangle .
$$

Applying the $\widetilde{\Delta}$ operator to $g$, we have

$$
\widetilde{\Delta} g=\widetilde{\Delta}\langle f(x)-\bar{f}(x), c\rangle=\widetilde{\Delta}\langle f(x), c\rangle-\tilde{\Delta}\langle\bar{f}(x), c\rangle=n\langle\eta, c\rangle-n\langle\bar{\eta}, c\rangle=0
$$

on $W \cup Q$. Since $g=0$ on $W$, then $g=0$ on $W \cup Q$ by the unique continuation principle of second order elliptic operators with $C^{\infty}$ coefficients ([3], [10], [11]). Hence $f(x)=\bar{f}(x)$ on $W \cup Q$ contradicting the maximality of $W$. Consequently, $W$ will be the whole of $M$ and so $f(x)=\bar{f}(x)$ for all points of $M$ and we have that $M$ and we have that $M$ is rigid which ends the proof of part (i).

Considering $m \in V_{2}$ where $\bar{A}=-A$, we apply $r$, given in Lemma 4.2 , to $H^{n+1}$ and carry out the proof taking

$$
g(x)=\langle f(x)-\tau f(\bar{x}), m\rangle
$$

as $m$ is invariant under $\tau$.

PROOF OF PART (ii). This part of the theorem may be considered as a corollary of part (i) in the following sense.

It has been proved in ([2], [5]) that if $M$ is compact and isometrically immersed in $H^{n+1}$, then there exists a point of $M$ all of whose sectional curvatures are positive. Applying part (i) we obtain the desired result.

PROOF OF PART (iii). In this case, as $\operatorname{tr} A \neq 0$ at $m$ and rank $A \neq 0$ everywhere on $M$, the same procedure can be followed but the required isometry of $H^{n+1}$ will be different from those of parts (i) and (ii).

ACKNOWLEDGEMENT. The author is grateful to the referee for his valuable suggestions.

\section{REFERENCES}

1. ABE, K. \& ERBACHER, J., Isometric immersions with the same Gauss map, Math. Ann. $\underline{215}(1975), 197-201$. 
2. AMARAL, L., Hypersurfaces in non-Euclidean space, Ph.D Thesis, Univ. California, Berkeley (1964).

3. ARONSZAJN, N., A unique continuation theorem for solutions for elliptic partial differential equations or inequalities of second order, J. Math. Pures \& Appl. 36 (1975), 235-249.

4. BELTAGY, M., Immersions into manifolds without conjugate points, Ph.D. Thesis, Durham Univ., U.K. (1982).

5. BELTAGY, M., A proof of L. Amaral's theorem,Ind. J. Pure \& Appl. Math. 14 (6) (1983), 703-706.

6. BeltaGY, M., A rigidity theorem in sphere, J. Math. \& Comp. Sci. (In Press).

7. DOCARMO, M.P. \& WARNER, F.W., Rigidity and convexity of hypersurfaces in sphere, J. Diff. Geo. 4 (1970), 133-144.

8. GOLDSTEIN, R.A. \& RYAN, P.J., Infinitesimal rigidity of submanifolds, J. Diff. Geo. 10 (1975), 49-60.

9. KOBAYASHI, S. \& NOMIZU, K., Foundations of Differential Geometry, Vol. I \& II, New York, Interscience Publishers (1963-1969).

10. MIZOHATA, S., Theory of partial differential equations, Cambridge Univ., 1973.

11. PROTTER, M., A unique continuation for elliptic equations, Trans. Amer. Math. Soc. 95 (1960), 81-91.

12. SACKSTEDER, R., The rigidity of hypersurfaces, J. Math. Mech. II (1962), 929-940.

13. SASAKI, S., A proof of the fundamental theorem of hypersurfaces in a space-form, Tensor, N.S. 24 (1972), 363-373.

14. SPIVAK, M., A Comprehensive Introduction to Differential Geometry, Vol. IV \& V, Publish or Perish, Inc., 1975. 


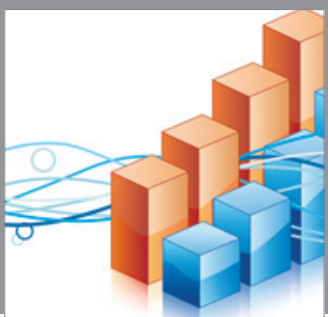

Advances in

Operations Research

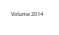

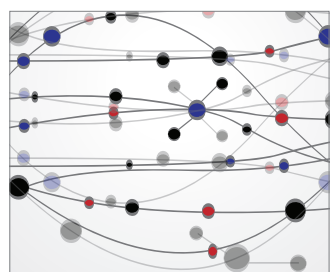

\section{The Scientific} World Journal
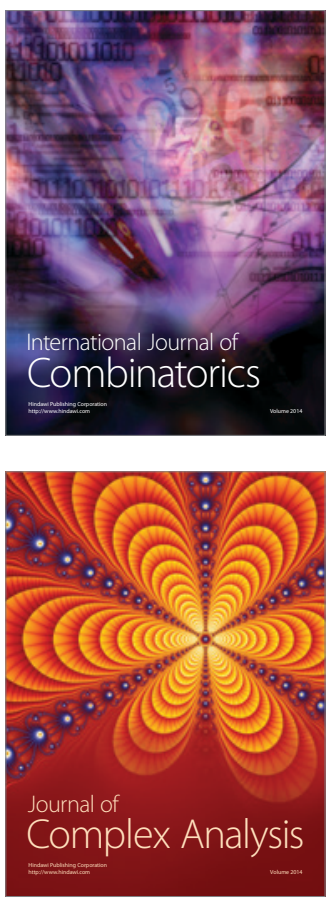

International Journal of

Mathematics and

Mathematical

Sciences
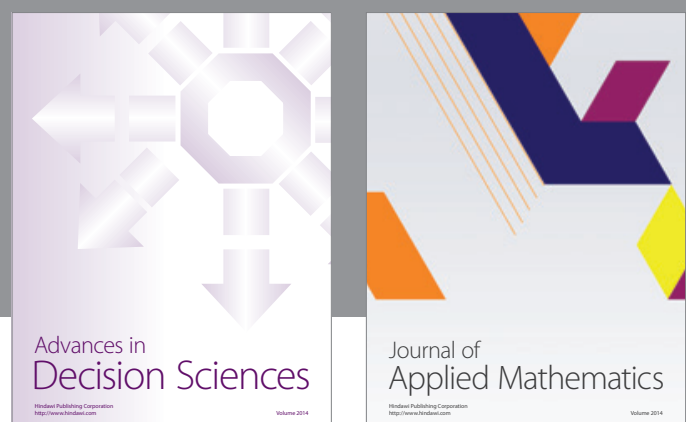

Journal of

Applied Mathematics
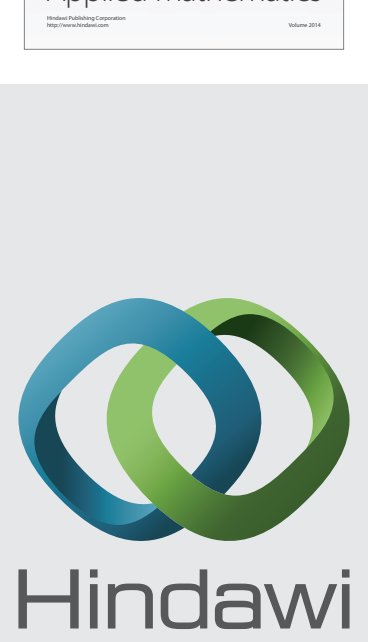

Submit your manuscripts at http://www.hindawi.com
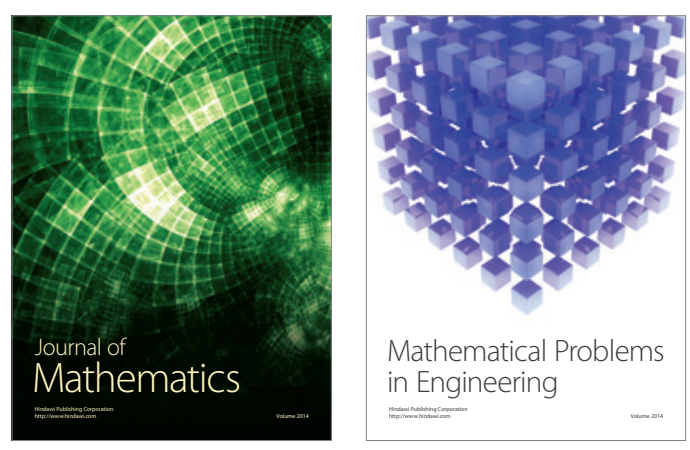

Mathematical Problems in Engineering
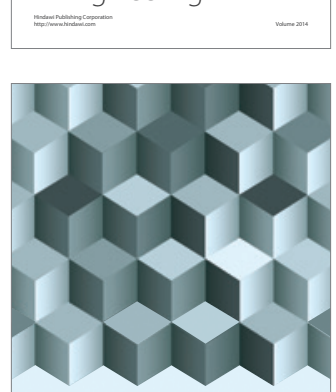

Journal of

Function Spaces
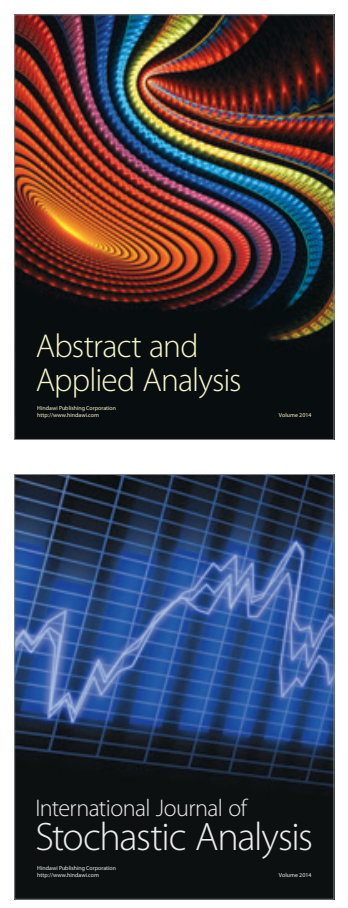

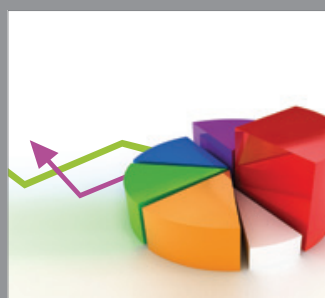

ournal of

Probability and Statistics

Promensencen
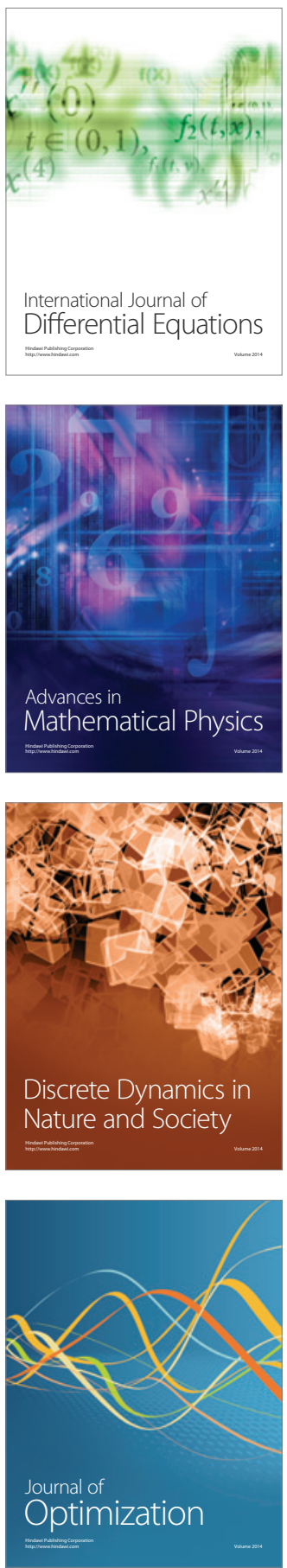